\title{
Solving Motion Law by Numerical Simulation on Bowl Seeding Transplanting Robot
}

\author{
Chunhui Qi, Jianping Hu, Jun Ma, and Jianbing Zhang \\ Key Laboratory of Modern Agricultural Equipment and Technology, \\ Ministry of Education \& Jiangsu Province, Jiangsu University, \\ High-tech Key Laboratory of Agricultural Equipment \& Intelligentization \\ of Jiangsu Province, Zhenjiang 212013, China \\ hujp@ujs.edu.cn
}

\begin{abstract}
The paper using the inverse kinematics theory of the parallel mechanism, studied the movable needs of the transplanting robot,deduced the inverse kinematics formula of transplanting robot. Considered the demand of the "fetch and plant" operations, made an important research on the two trajectory planning methods of the moving platform: quintic and septic polynomial motion laws, acquired their variation rules of displacement, velocity, acceleration and saltus by using matlab software, and to make choice. After selected the law of motion equation, through numerical simulation of inverse kinematics by matlab, acquired kinematic parameters angular speed and acceleration of the two active motors, can be used as the priority basis for control the movement of the manipulator.
\end{abstract}

Keywords: Parallel mechanism, transplanting bowl seeding, trajectory planning, inverse position, numerical simulation.

\section{$1 \quad$ Preface}

Pot seeding technology has many advantages, such as reduce man-hour, raise working efficiency and protect the agricultural ecology environment, and it has been used in production of vegetable and flower widely, transplanting bowl seeding is an important link. Although transplanting bowl seeding is easy, it needs amount of hand labour, the average speed of artificially transplanting is 800-1000 trees per hour, and the workers work continuously will get tired easily, a low production efficiency, and hard to realize large-scale operations. So, to research high-speed automatic bowl seedling transplanting machine is particularly important, it has important significance in improve labor productivity, reduce the labor intensity, promote potted tray seeding technology development. Currently, transplanting machines with the industrial robot as body have many shortcomings, such as many freedoms, complicated structure and so forth, have many difficults to fit for greenhouse automatic assembly line working facilities, so the type of automatic potted tray seedling transplanting machine fails to get promotion.

The paper will put forward a Two-DOF parallel transplanting robot of independent mechanical and electrical system, as shown in Figure 1, the mechanism consists of a five-bar parallel mechanism, two groups parallelogram chain and a moving platform, 
driven by the active motors, pneuncatic manipulator could achieve two-dimensional translational. Due to the driving motors fixed on the frame, driven arms are made into light bars, so could make the moving platform achieve a high speed and acceleration, to meet bowl seedlings transplanting demand of high speed and short to medium distance.

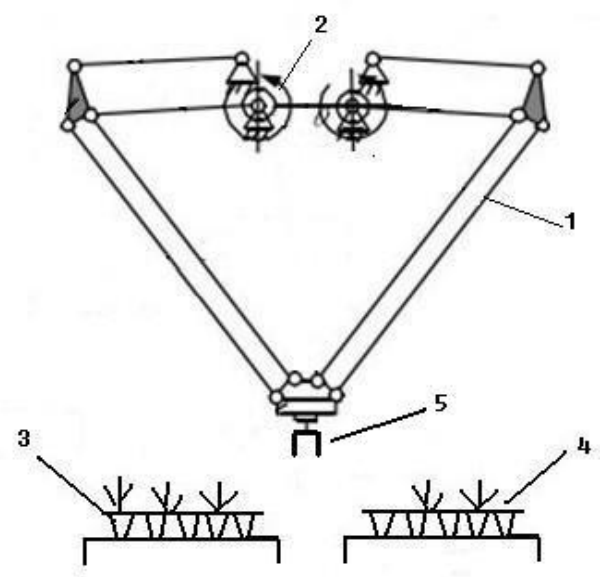

Fig. 1. Schematic diagram of transplanting robot 1. parallel mechanism 2. motor 3. seeding tray 4. plant seeding tray 5. manipulator

\section{Select Bowl Seeding Transplanting Robot Motion Law}

\subsection{Motion Law Requirement of Two-DOF Parallel Transplanting Robot}

Two-DOF parallel mechanism used to pick up and placed the object, its main job is pick up object in the initial position, then move to the target position placed the object, kinematic sketch is shown in Figure 2.

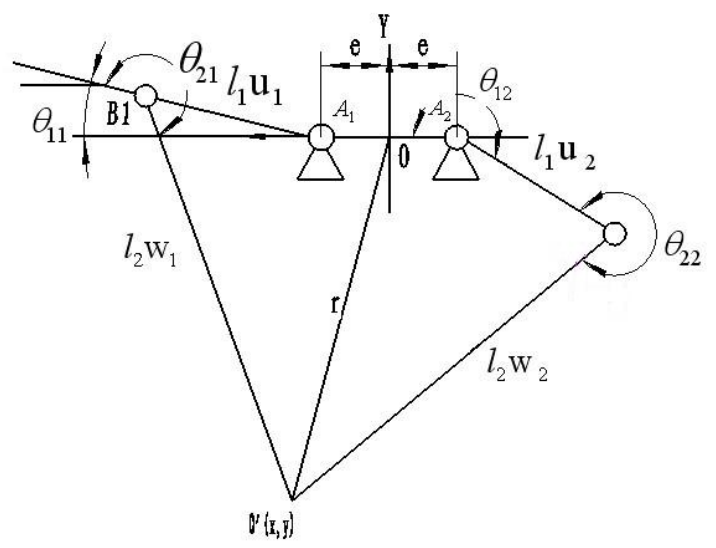

Fig. 2. Kinematic sketch 
Working space of the mechanism is $600 * 150 \mathrm{~mm}$, maximum speed of the manipulator is $4.5 \mathrm{~m} / \mathrm{s}$, the maximum acceleration is $60 \mathrm{~m} / \mathrm{s} 2$. Maximum load weight is $0.3 \mathrm{Kg}$, the average frequency of transplanting reach 100 times/minute.

To make the moving platform under the condition of the maximum acceleration allowed, the fastest way to fetch seedling from a certainl position of the seedling tray, then set the seeding to the corresponding position of the plant seedling tray, must be based on position, velocity and acceleration to plan trajectory.

\subsection{Motion Trajectory Planning on Transplanting Manipulator}

In the process of transplanting bowl seedings, manipulator required fetch seeding and plant seeding the two motion process for each seeding transplanting. While each process, the manipulator would pass through rising, translation, decline three stages. Transplanting motion requires the manipulator on the rise and decline section must keep a certain distance of straight-line movement, as $\mathrm{AB}$ and $\mathrm{CD}$ section shown in Figure 3:

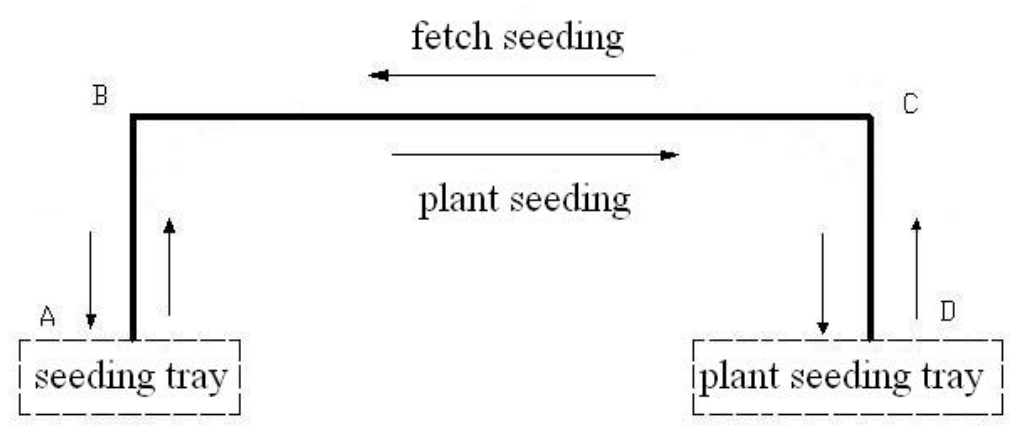

Fig. 3. Manipulator trajectory of transplanting process

For each straight line track, set motion law of the manipulator is cease-speed up- speed down-cease. So to achieve this rule, must meet the following points:

1) Moving platform velocity and acceleration at the start and end of the trajectory is zero;

2) First and second derivatives of displacement motion law must be continuous;

3) The third-order derivative of the displacement motion law $f$ (the derivative of acceleration) is limited; otherwise it will cause shock; to ensure that the manipulator avoid to be impacted.

\subsection{Mathematical Representation of the Two Motion Laws}

In less than a given maximum acceleration condition, base on the movement theory, there are many motion laws to meet the above requirements, such as sine law, cycloidal law, trapezoidal law, quintic polynomial motion law, septic polynomial motion law and 
so on. Because quintic and septic polynomial are more likely to achieve above motion requirements accurately, therefor, only conduct analysis on them. They are under the condition of known the displacement $S$ and the maximum acceleration amax , the calculation formula is as follows:

1) Quintic polynomial motion law

Displacement equation

$$
\mathrm{s}=\frac{\mathrm{a}_{\max }}{5.7735} \mathrm{~T}^{2}\left(10\left(\frac{t}{T}\right)^{3}-15\left(\frac{t}{T}\right)^{4}+6\left(\frac{t}{T}\right)^{5}\right)
$$

Derivative of (1), acquire velocity, acceleration and saltus equation

$$
\begin{gathered}
\mathrm{v}=\frac{d s}{d t}=\frac{30 \mathrm{a}_{\max }}{5.7735} \mathrm{~T}\left(\left(\frac{t}{T}\right)^{2}-2\left(\frac{t}{T}\right)^{3}+\left(\frac{t}{T}\right)^{4}\right) \\
\mathrm{a}=\frac{d^{2} s}{d t^{2}}=\frac{60 \mathrm{a}_{\max }}{5.7735}\left(\left(\frac{t}{T}\right)-3\left(\frac{t}{T}\right)^{2}+2\left(\frac{t}{T}\right)^{3}\right) \\
\mathrm{j}=\frac{d^{3} s}{d t^{3}}=\frac{60 \mathrm{a}_{\max }}{5.7735}\left(\frac{1}{T}\right)\left(1-6\left(\frac{t}{T}\right)+6\left(\frac{t}{T}\right)^{2}\right)
\end{gathered}
$$

Total motion time $\mathrm{T}$

$$
T=\sqrt{\frac{5.7735 S}{\mathrm{a}_{\max }}}
$$

2) Septic polynomial motion law

Displacement equation

$$
\mathrm{s}=\frac{\mathrm{a}_{\max }}{7.51} \mathrm{~T}^{2}\left(35\left(\frac{t}{T}\right)^{4}-84\left(\frac{t}{T}\right)^{5}+70\left(\frac{t}{T}\right)^{6}-20\left(\frac{t}{T}\right)^{7}\right)
$$

Derivative of (6) , acquire velocity, acceleration and saltus equation

$$
\begin{gathered}
\mathrm{v}=\frac{d s}{d t}=\frac{140 \mathrm{a}_{\max }}{7.51} \mathrm{~T}\left(\left(\frac{t}{T}\right)^{3}-3\left(\frac{t}{T}\right)^{4}+3\left(\frac{t}{T}\right)^{5}-\left(\frac{t}{T}\right)^{6}\right) \\
\mathrm{a}=\frac{d^{2} s}{d t^{2}}=\frac{420 \mathrm{a}_{\max }}{7.51}\left(\left(\frac{t}{T}\right)^{2}-4\left(\frac{t}{T}\right)^{3}+5\left(\frac{t}{T}\right)^{4}-2\left(\frac{t}{T}\right)^{5}\right)
\end{gathered}
$$




$$
\mathrm{j}=\frac{d^{3} s}{d t^{3}}=\frac{840 \mathrm{a}_{\max }}{7.51}\left(\frac{1}{T}\right)\left(\left(\frac{t}{T}\right)-6\left(\frac{t}{T}\right)^{2}+10\left(\frac{t}{T}\right)^{3}-5\left(\frac{t}{T}\right)^{4}\right)
$$

Total motion time $\mathrm{T}$

$$
T=\sqrt{\frac{7.51 S}{\mathrm{a}_{\max }}}
$$

\subsection{Simulation Analysis of the Two Motion Laws}

Applied Mathematics software matlab, obtain the following motion law of Figure 4 to Figure 7.

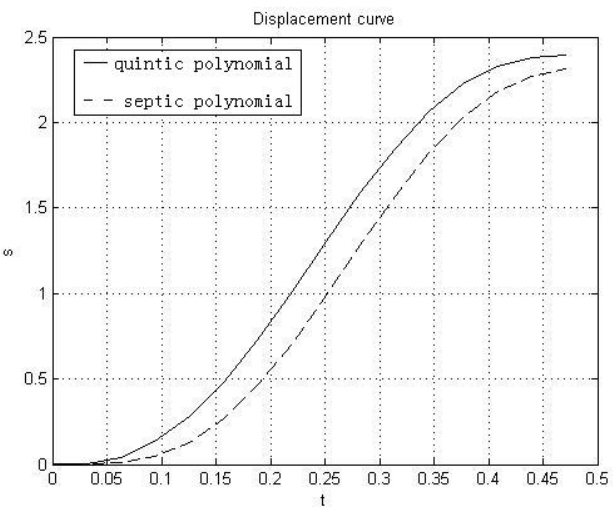

Fig. 4. Displacement curve

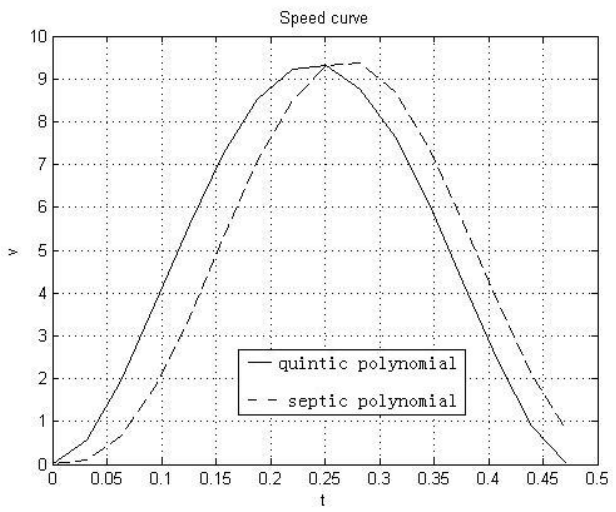

Fig. 5. Speed curve 


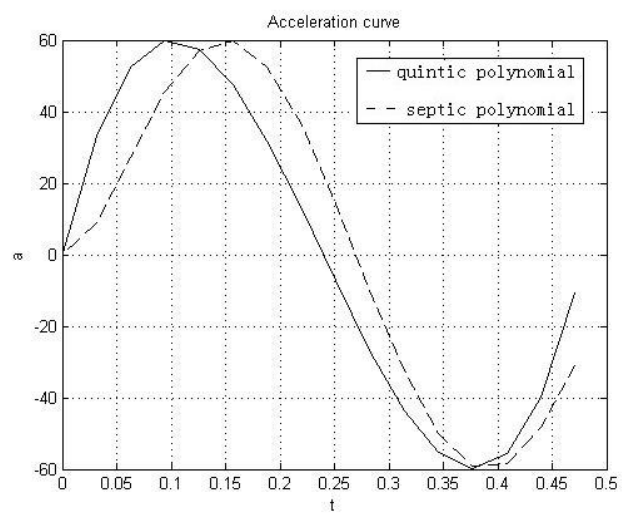

Fig. 6. Acceleration curve

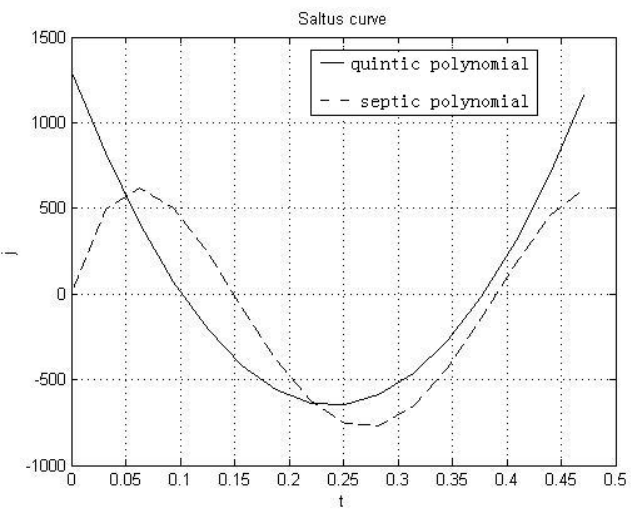

Fig. 7. Saltus curve

From the above charts we can see, quintic polynomial motion law arrive the maximum displacement, velocity, acceleration, spent shorter time, more stable saltus, indicating the saltus of acceleration is smaller; under the condition of the motion laws have the same size of displacement and the maximum acceleration, quintic polynomial motion law uses a shorter time of $0.07 \mathrm{~s}$.

In the case of ensure the moving platform smooth operation, select the quintic polynomial motion law as the trajectory function, can greatly reduce the time used in transplanting, improve transplanting efficiency. 


\section{Solving the Active Motor Motion Laws of the High-Speed Parallel Transplanting Robot}

\subsection{Position Inverse Model}

Refer to Figure 2, in fixed reference coordinate system $\mathrm{O}-\mathrm{xy}$, establish equation:

$$
r-\operatorname{sgn}(i) e e_{1}-l_{1} u_{i}=l_{2} w_{i}
$$

and, $\quad u_{i}=\left(\cos \theta_{1 i} \sin \theta_{1 i}\right)^{T}, w_{i}=\left(\cos \theta_{2 i} \sin \theta_{2 i}\right)^{T}$,

$$
e_{1}=\left(\begin{array}{ll}
1 & 0
\end{array}\right)^{T}, \operatorname{sgn}(i)=\left\{\begin{array}{cc}
1 & i=1 \\
-1 & i=2
\end{array}\right.
$$

so: $A_{i} \sin \theta_{1 i}+B_{i} \cos \theta_{l i}+C_{i}=0$

$$
\theta_{1 i}=2 \operatorname{artan} \frac{-A_{i}+\operatorname{sgn}(i) \sqrt{A_{i}^{2}-C_{i}^{2}+B_{i}^{2}}}{C_{i}-B_{i}}
$$

among that, $\quad A_{i}=-2 l_{1} y$

$$
\begin{aligned}
& B_{i}=-2 l_{l}(x-\operatorname{sgn}(i) e) \\
& C_{i}=x^{2}+y^{2}+e^{2}+l_{1}^{2}-l_{2}^{2}-2 \operatorname{sgn}(i) e x
\end{aligned}
$$

\subsection{Velocity, Acceleration Model}

First and second order derivation of time for formula (11) , can get system speed and acceleration model:

$$
\begin{gathered}
\dot{\theta}_{1}=\frac{1}{l_{1}} J v \\
J a=l_{1} \ddot{\theta}_{1}-l_{1} f\left(\dot{\theta}_{1}\right)
\end{gathered}
$$

In the formula, $v$ and $a$ are speed and acceleration of $O$, speed Jacobian matrix i:

$$
J=\frac{1}{l_{1}}\left[\begin{array}{cc}
w_{1} & w_{2} \\
w^{T}{ }_{1} Q u_{1} & w^{T}{ }_{2} Q u_{2}
\end{array}\right]^{T}
$$


110 C. Qi et al.

$$
Q=\left[\begin{array}{cc}
0 & -1 \\
1 & 0
\end{array}\right]^{T}
$$

\subsection{Numerical Simulation Curve of the Two Active Motors}

Set the manipulator horizontal moving distance $600 \mathrm{~mm}$, vertical moving distance $150 \mathrm{~mm}$, in the condition of a given mechanism parameter, inverse simulation by software matlab, acquire the following curves of the active motors:

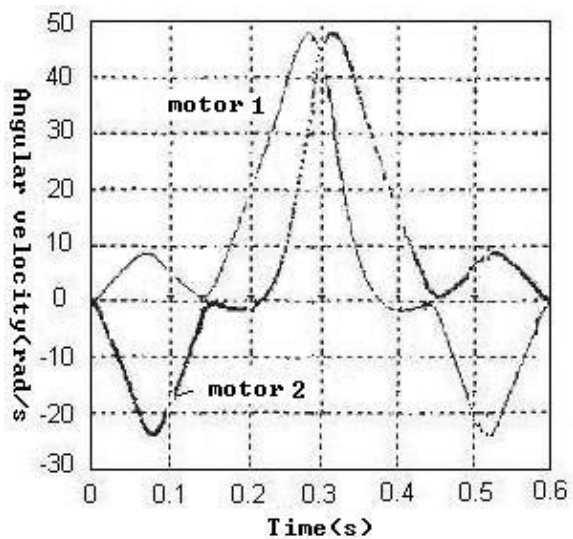

Fig. 8. Angular velocity curve

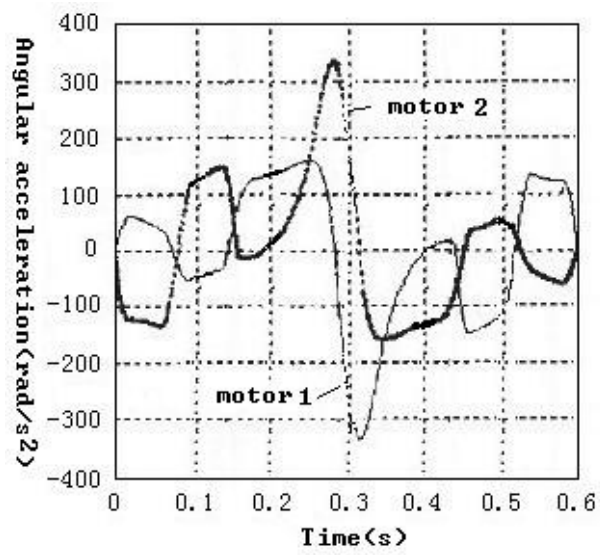

Fig. 9. Angle acceleration curve

Obtain the motors numerical parameters from above figures, provided theoretical basis for control the manipulator and optimal design the physical prototype. 


\section{Conclusion}

1) Using the inverse kinematics theory of the parallel mechanism, studied the movable needs of the transplanting robot, deduced the inverse kinematics formula of transplanting robot.

2) Motion trajectory of transplanting robot was planned, conducted numerical analysis for quintic and septic polynomial by matlab software, selected the better motion polynomial.

3) Apply quintic polynomial motion law, under a certain condition of motor motion law, the operating cycle to be shorter, meet the requirement of high-speed robot for transplanting, can reduce the transplanting time of $0.07 \mathrm{~s}$, improve the transplanting efficiency.

Acknowledgements. This work was financially supported by Three Engineering of Agricultural Machinery, Jiangsu province(NJ2009-41), by Qing Lan Project of Jiangsu Province (Su teacher (2010)no.27) and the Priority Academic Program Development of Jiangsu Higher Education Institutions (Su financial teacher(2011)no.8).

\section{References}

1. Choi, W.C., Kim, D.C., Ryu, I.H., et al.: Development of a seeding pick-up device for vegetable transplanters. Transactions of the ASAE 45(1), 13-19 (2002)

2. Kutz, L., Miles, G.E.: Robotic transplanting of bedding plants. Transactions of the ASAE 30(3), 586-590 (1987)

3. Huang, T., Li, Z.X., Li, M., et al.: Conceptual design and dimensional synthesis of a novel 2-DOF translational parallel robot for pick-and-place operations. ASME Journal of Mechanical Design 126(5), 449-455 (2004)

4. Borubakis, N., Kydonas, G.: An Autonomous Hybrid Robot:Walking and Climbing. IEEE Robotics and Automation Magazine 11(6), 52-59 (1998)

5. Hiller, M., Fang, S.Q., Mielczarek, S.: Design Analysis and Realization of Parallel Tendon-based Manipulators. Mechanism and Machine Theory 40(4), 429-455 (2005)

6. Ryu, K.H., Kim, G., Han, J.S.: Development of a robotic transplanter for bedding plants. Journal of Agricultural Engineering Research 78(2), 141-146 (2001)

7. Guo, Z., Li, L., Sun, S.: Trajectory Planning and Simulation on 3-RPC Parallel Mechanism. The Chinese Mechanical Engineering 18(5), 1036-1038 (2007)

8. Ting, K.C.: Automate flexibly with robots. Greenhouse Grower 5(11), 24-28 (1987)

9. Youcef, T.K., Kuo, A.T.Y.: High-speed trajectory control of a direct-drive manipulator. IEEE Transactions on Robotics and Automation 2(1), 102-108 (1993)

10. Arai, T., Funabashi, H., Nakamura, Y., Takeda, Y., Koseki, Y.: High speed and high precision parallelmechanism. In: Proceedings of the 1997 IEEE/RSJ International Conference on Intelligent Robots and Systems, vol. 3(7-11), pp. 1624-1629 (1997)

11. Ji, Z.M.: Dynamics decomposition for Stewart platforms. J. of Mechanical Design 116, 67-69 (1994)

12. Lee, K.M., Shan, D.K.: Dynamic analysis of a Three-Degrees-Freedom In-Parallel actuated manipulator. IEEE Transactions On Robotics and Automation 4(3), 361-367 (1988) 